\title{
SPECTRALLY RESOLVED PURE ROTATIONAL LINES OF WATER IN PROTOPLANETARY DISKS
}

\author{
Klaus M. Pontoppidan ${ }^{1}$, Colette Salyk ${ }^{1,2}$, Geoffrey A. Blake ${ }^{1}$, and Hans Ulrich KäUfl ${ }^{3}$ \\ ${ }^{1}$ California Institute of Technology, Division of Geological and Planetary Sciences, MS 150-21, Pasadena, CA 91125, USA; pontoppi@gps.caltech.edu \\ ${ }^{2}$ Department of Astronomy, The University of Texas at Austin, 1 University Station C1400, Austin, TX 78712, USA \\ ${ }^{3}$ European Southern Observatory, Karl-Schwarzschild-Strasse 2, 85748 Garching bei München, Germany \\ Received 2010 August 6; accepted 2010 September 16; published 2010 September 29
}

\begin{abstract}
We present ground-based high-resolution $N$-band spectra $\left(\Delta v=15 \mathrm{~km} \mathrm{~s}^{-1}\right)$ of pure rotational lines of water vapor in two protoplanetary disks surrounding the pre-main-sequence stars AS 205N and RNO 90, selected based on detections of rotational water lines by the Spitzer InfraRed Spectrograph. Using VISIR on the Very Large Telescope, we spectrally resolve individual lines and show that they have widths of $30-60 \mathrm{~km} \mathrm{~s}^{-1}$, consistent with an origin in Keplerian disks at radii of $\sim 1 \mathrm{AU}$. The water lines have similar widths to those of the $\mathrm{CO}$ at $4.67 \mu \mathrm{m}$, indicating that the mid-infrared water lines trace similar radii. The rotational temperatures of the water are 540 and $600 \mathrm{~K}$ in the two disks, respectively. However, the line ratios show evidence of non-LTE excitation, with low-excitation line fluxes being overpredicted by two-dimensional disk LTE models. Due to the limited number of observed lines and the non-LTE line ratios, an accurate measure of the water ortho/para $(\mathrm{O} / \mathrm{P})$ ratio is not available, but a best estimate for AS $205 \mathrm{~N}$ is $\mathrm{O} / \mathrm{P}=4.5 \pm 1.0$, apparently ruling out a low-temperature origin of the water. The spectra demonstrate that high-resolution spectroscopy of rotational water lines is feasible from the ground, and further that ground-based high-resolution spectroscopy is likely to significantly improve our understanding of the inner disk chemistry revealed by recent Spitzer observations.
\end{abstract}

Key words: astrochemistry - protoplanetary disks - planetary systems

Online-only material: color figures

\section{INTRODUCTION}

The study of the dynamics and chemistry of the inner $(R<10 \mathrm{AU})$ regions of disks around young pre-main-sequence stars is a field in rapid growth, to a large extent thanks to the availability of sensitive infrared instrumentation, including the InfraRed Spectrograph on the Spitzer Space Telescope. The molecular gas at these radii plays a key role in the process of planet formation, not only as a necessary reservoir for the formation of gas giants, but also in the generation and eventual delivery of volatile molecular species to terrestrial planets.

Recent observations have demonstrated that the mid-infrared wavelength range in protoplanetary disks contains a forest of molecular emission lines, many of them due to pure rotational transitions of water vapor (Carr \& Najita 2008; Salyk et al. 2008; Pascucci et al. 2009; Pontoppidan et al. 2010a). While Carr et al. (2004) detected and spectrally resolved rovibrational water lines at $2.3 \mu \mathrm{m}$ and Salyk et al. (2008) at $3 \mu \mathrm{m}$ from protoplanetary disks, the observations of the mid-infrared pure rotational lines were all carried out at $R=\lambda / \Delta \lambda \leqslant 600$, resulting in unresolved and blended line spectra. The rovibrational $2-3 \mu \mathrm{m}$ lines have much higher excitation temperatures and may not probe the same gas as the mid-infrared rotational lines. Further, they also suffer from significant line blending, making it difficult to study the details of the excitation structure. In this Letter, we demonstrate that high-resolution spectroscopy of pure rotational transitions of water in the atmospheric $N$-band window is possible. The rotational water lines observable from the ground have excitation temperatures of 3000-6000 K, directly comparable to those of the well-studied CO rovibrational lines in the $4.7 \mu \mathrm{m}$ fundamental band (Najita et al. 2003; Blake \& Boogert 2004; Brittain et al. 2007; Pontoppidan et al. 2008; Salyk et al. 2009), allowing for more accurate relative abundance measurements.
Further, the lines are well separated from one another and do not suffer from blending with other species.

In this Letter, we present high-resolution $(R \sim 20,000) \mathrm{N}$ band spectra of AS 205N and RNO 90, disks known to have strong water vapor emission (Salyk et al. 2008; Pontoppidan et al. 2010a), obtained using the Very Large Telescope Imager and Spectrometer for the mid-InfraRed (VISIR; Lagage et al. 2004). Both disks are likely part of the Ophiuchus star-forming cloud at a distance of $120 \mathrm{pc}$ (Loinard et al. 2008). With stellar luminosities of 4.0 and $3.5 L_{\odot}$ (Chen et al. 1995) and spectral types of K5 and G5, the central stars have likely masses close to $1 M_{\odot}$ (e.g., Siess et al. 1997; Salyk et al. 2008; Pontoppidan et al. 2010b). RNO 90 in particular, being a single star, may be a fairly close analog to the young Sun, while AS $205 \mathrm{~N}$ is the primary of a 1".3 binary (160 AU).

\section{OBSERVATIONS}

Six water lines with excitation energies varying from 3300 to $5800 \mathrm{~K}$ were selected using the list of Pontoppidan et al. (2009) in combination with the limitations imposed by the availability of filters on VISIR. While telluric water generates highly pressure-broadened absorption, we find that even from a relatively low site, such as Paranal Observatory $(2635 \mathrm{~m})$, the atmospheric transmission for all our targeted lines is $>30 \%$ and often significantly better.

Spectra centered on 12.405 and $12.454 \mu \mathrm{m}$ were observed using the cross-dispersed mode of VISIR, with a chop throw of $8^{\prime \prime}$. In this mode, one source position is off the slit because the minimum chop throw is larger than the slit length. Additional settings centered on 12.829 and $12.893 \mu \mathrm{m}$ were observed using the long-slit mode, in which all source positions are on slit, doubling the effective exposure time. The effective on-source 
exposure times were $1250 \mathrm{~s}$ and $2500 \mathrm{~s}$ for the cross-dispersed and long-slit modes for AS 205N, respectively. For RNO 90, these exposure times were doubled. All observations were carried out using the 0.75 slit during good seeing conditions, specifically ensuring that components of the AS 205 binary are well separated. AS 205N was observed on 2009 August 5 and 6, while RNO 90 was observed on separate nights between 2009 August 9 and 2009 September 11. The spectral images were coadded using the ESO VISIR pipeline version 3.2.2. Chopping parallel to the slit removes most of the sky background, but a significant residual, especially from rapidly varying atmospheric water lines, is still visible. These residuals were removed by subtracting the median of each row (in the cross-dispersion direction) from the spectral image. The one-dimensional spectra were generated using optimal extraction (Horne 1986). The wavelength calibration from the ESO pipeline was used and is computed by cross-correlating the sky emission spectrum with an atmospheric model generated using HITRAN (Rothman et al. 2005).

The spectral response function and atmospheric transmission spectrum were measured using observations of a bright infrared excess source known to not display strong water emission at $12 \mu \mathrm{m}$ in the survey of Pontoppidan et al. (2010a), specifically the bright Herbig Ae/Be star HD150193. Ideally, a hot star without excess should be used, but no such source is sufficiently bright in the $N$ band. One could use a later type star, but in that case photospheric absorption lines will be present that may be difficult to remove. Hence, the choice was made to use an excess source dominated by continuum emission and with no apparent lines. Corrections for small shifts (of order a few pixels) between the source spectrum and that of the standard as well as a correction for differences in water column densities using a simple Lambert-Beer law were made to minimize the telluric residuals. The absolute fluxes were scaled to match those of the Spitzer spectra in Pontoppidan et al. (2010a).

For comparison with the water lines, we use archival $M$-band high-resolution $(R=100,000)$ spectra of the CO rovibrational fundamental $(\Delta v=1)$ lines observed with CRIRES. The CO lines have roughly the same upper level energies as the $N$-band rotational water lines. The CRIRES data set will be described in greater detail in a spectro-astrometric survey (Pontoppidan et al. 2010b). It was processed following the procedures of Pontoppidan et al. (2008). Finally, we extracted an isolated lower energy line for comparison from the Pontoppidan et al. (2010a) Spitzer spectra. The low-resolution $(R=600)$ Spitzer spectra do not allow for the measurement of many unblended lines, but there are a few exceptions, one of them being the $10_{47} \rightarrow 9_{36}$ ortho line at $33.5 \mu \mathrm{m}$.

\section{RESULTS}

The reduced VISIR spectra of AS 205N and RNO 90 are shown in Figures 1 and 2, respectively. For AS 205N, we detect five out of the six targeted lines, while three lines are detected for RNO 90. Integrated line fluxes are given in Table 1.

The lines, where detected, are spectrally resolved, with line widths of $\sim 30 \mathrm{~km} \mathrm{~s}^{-1}$ for AS $205 \mathrm{~N}$ and $\sim 60 \mathrm{~km} \mathrm{~s}^{-1}$ for RNO 90. In the figures, the water lines are compared to the $\mathrm{CO}$ $\Delta v=1$ line shape. While variations are apparent for AS $205 \mathrm{~N}$, these differences are relatively minor, with an FWHM of $20-35 \mathrm{~km} \mathrm{~s}^{-1}$ similar to or slightly less than those of CO. This indicates that the water lines are formed at similar or slightly larger disk radii. The high energy lines $\left(17_{43} \rightarrow 16_{34}\right.$, $16_{313} \rightarrow 15_{214}$ ) of AS $205 \mathrm{~N}$ show indications of being flat-
Table 1

$\mathrm{H}_{2} \mathrm{O}$ Line Fluxes

\begin{tabular}{lcccc}
\hline \hline \multicolumn{1}{c}{ Line } & $\begin{array}{c}\text { Wavelength } \\
(\mu \mathrm{m})\end{array}$ & $\begin{array}{c}E_{\text {upper }} \\
(\mathrm{K})\end{array}$ & AS 205N & RNO 90 \\
\hline $17_{413} \rightarrow 16_{314}(\mathrm{o})$ & 12.396 & 5746 & $4.8 \pm 0.2$ & $0.7 \pm 0.08$ \\
$16_{313} \rightarrow 15_{214}(\mathrm{p})$ & 12.407 & 4915 & $2.3 \pm 0.2$ & $0.9 \pm 0.11$ \\
$13_{76} \rightarrow 12_{49}(\mathrm{o})$ & 12.453 & 4187 & $10.4 \pm 0.2$ & $1.0 \pm 0.14$ \\
$11_{83} \rightarrow 10_{56}(\mathrm{o})$ & 12.445 & 3606 & $11.4 \pm 0.3$ & $<0.3$ \\
$12_{57} \rightarrow 11_{210}(\mathrm{p})$ & 12.893 & 3290 & $1.9 \pm 0.2$ & $<0.3$ \\
$10_{82} \rightarrow 9_{55}(\mathrm{p})$ & 12.832 & 3223 & $<0.4$ & $<0.2$ \\
$10_{47} \rightarrow 9_{36}(\mathrm{o})$ & 33.501 & 2260 & $1.9 \pm 0.2$ & $<0.3$ \\
\hline
\end{tabular}

Note. ${ }^{a} 10^{-14} \mathrm{erg} \mathrm{cm}^{-2} \mathrm{~s}^{-1}$.

topped, while the lower excitation lines are single peaked, as is CO. All three detected RNO 90 lines are consistent with being double peaked, also matching the corresponding $\mathrm{CO}$ lines. The double peak is apparent when the three detected line profiles are averaged. We interpret this as evidence for a line origin in a Keplerian disk.

In order to estimate the properties of the emitting gas (rotational temperatures, radial distribution, column densities, and abundances), we model the spectra using the two-dimensional ray tracer RADLite (Pontoppidan et al. 2009). We also construct rotation diagrams (Figure 3), which are designed to allow for easy comparison with a model consisting of a single-temperature slab of gas, and enable a discussion of the excitation and column density, in an average sense, of the observed species; LTE level populations and optically thin emission predict that line fluxes are arranged along a straight line in the rotation diagram. Here, we demonstrate that there are departures from the basic slab model assumptions of LTE, and that the assumption that all lines are formed in the same solid angle does not hold for water. Specifically, the slab model predicts significantly brighter $10_{82} \rightarrow 9_{55}$ and $12_{57} \rightarrow 11_{210}$ emission, by at least factors of $5-10$, than what is observed for AS $205 \mathrm{~N}$. The upper limits on the RNO 90 lines are not strongly constraining, and the rotation diagram for this disk is roughly consistent with the slab model in the $N$ band, while the Spitzer $33.5 \mu \mathrm{m}$ line is underluminous. The lower energy Spitzer line thus confirms the $N$-band turnover at lower energies in the rotation diagram for AS $205 \mathrm{~N}$. We stress that these are differences that are not apparent from the low-resolution Spitzer spectra alone. A linear regression of the VISIR lines on the linear part of the rotation diagram $\left(E_{\text {upper }}>3500 \mathrm{~K}\right)$ yields rotational temperatures of 540 and $600 \mathrm{~K}$ for AS $205 \mathrm{~N}$ and RNO 90, respectively, for optically thin emission.

The AS $205 \mathrm{~N}$ temperature can be compared to the $1000 \mathrm{~K}$ required to fit the $3 \mu \mathrm{m}$ rovibrational lines (Salyk et al. 2008), indicating that the rotational lines indeed trace cooler gas. The selection of lines includes ortho and para transitions. The assumption that the $N$-band lines should form a straight line in the rotation diagram allows an estimate to be made of the $\mathrm{O} / \mathrm{P}$ ratio. The paucity of lines, and the presence of clear departures from LTE and/or slab geometry, unfortunately makes such an estimate uncertain. Nevertheless, scaling the three ortho lines for AS $205 \mathrm{~N}$ with energies between 3500 and $6000 \mathrm{~K}$ to an optimal alignment with the two detected para lines results in an apparent $\mathrm{O} / \mathrm{P}$ ratio of $4.5 \pm 1.0$ for $\mathrm{AS} 205 \mathrm{~N}$. The ratio is higher than 3 because the para lines are placed below a straight line defined by the three ortho lines, but by less than $2 \sigma$. While this measurement is certainly affected by the caveats discussed above, we interpret this as indicative of a warm gas-phase 


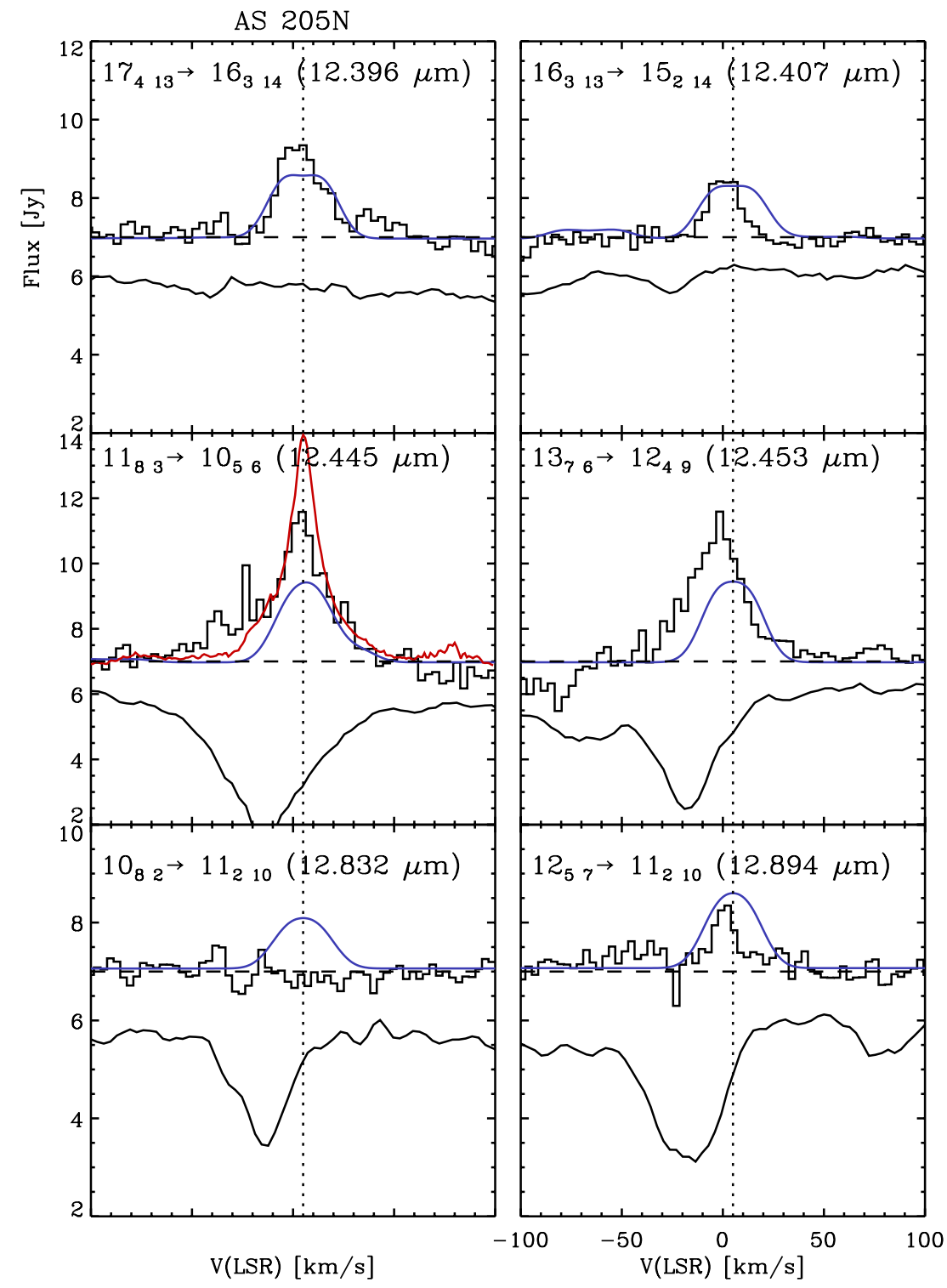

Figure 1. VISIR spectra of rotational water lines observed in AS $205 \mathrm{~N}$. The curves below the spectra show the standard star spectra, which are a combination of the spectral response function and the atmospheric transmission. The velocity range of the spectra is referenced to the local standard of rest. The red curve is the CO rovibrational $(\Delta v=1)$ line shape $(J<8)$, as observed with CRIRES (Pontoppidan et al. 2010b), whose center defines the vertical dotted line. The blue curves are lines calculated using an LTE two-dimensional disk model (see the text for details).

(A color version of this figure is available in the online journal.)

(equilibrium) chemistry, as opposed to water formed in a lowtemperature environment $(<60 \mathrm{~K})$ via grain-surface chemistry, which would produce $\mathrm{O} / \mathrm{P}$ ratios $<3$. Indeed, cometary water has $\mathrm{O} / \mathrm{P}$ ratios corresponding to spin temperatures of $\sim 30 \mathrm{~K}$ (Crovisier et al. 1997; Kawakita et al. 2004), and optical depth effects would drive the estimated $\mathrm{O} / \mathrm{P}$ values lower.

Since the lines are now spectrally resolved, the emitting area and hence the column density of the emitting gas can be directly estimated by converting the line width to a physical location in a Keplerian disk. The inclination of the RNO 90 disk is $\sim 37^{\circ}$ (Pontoppidan et al. 2010b) and the star has a mass of $\sim 0.9 M_{\odot}$ (Andrews et al. 2009). The RNO 90 water lines extend to $\sim 30 \mathrm{~km} \mathrm{~s}^{-1}$, corresponding to an inner water line emitting radius of 0.45 AU. Similarly, for AS $205 \mathrm{~N}$ the line width at zero flux corresponds to an inner emitting radius of $0.1-0.4 \mathrm{AU}$, for the $11_{83} \rightarrow 10_{56}$ and $17_{413} \rightarrow 16_{314}$ lines, respectively, assuming Keplerian flow, a disk inclination of $25^{\circ}$ and a stellar mass of $1 M_{\odot}$ (Andrews et al. 2009). The effective outer radii of the emitting areas are determined by generating two-dimensional models for the lines with RADLite, assuming LTE level populations. The model uses a simple parameterized flared disk structure (Dullemond et al. 2001), with inner radii fixed according to the observed line widths. The water abundance is set to $2.6 \times 10^{-4}$ per $\mathrm{H}$ and the disk surface gas-to-dust ratio set to $1.28 \times 10^{4}$, simulating significant dust settling, as discussed in Meijerink et al. (2009). Finally, the stellar luminosities were adjusted to match the dust continuum at $12.5 \mu \mathrm{m}$. The resulting line strengths and profiles match well to those of the detected $N$-band lines with $E>4000 \mathrm{~K}$, as was already suggested by the rotation diagram (see Figures 1 and 2). Figure 4 shows the corresponding line surface brightness from the RADLite model, demonstrating that the RNO 90 emission is dominated by radii from 0.4 to $1.0 \mathrm{AU}\left(\sim 2.6 \mathrm{AU}^{2}\right)$, but with the emitting area changing by a factor of $2-3$, depending on the 


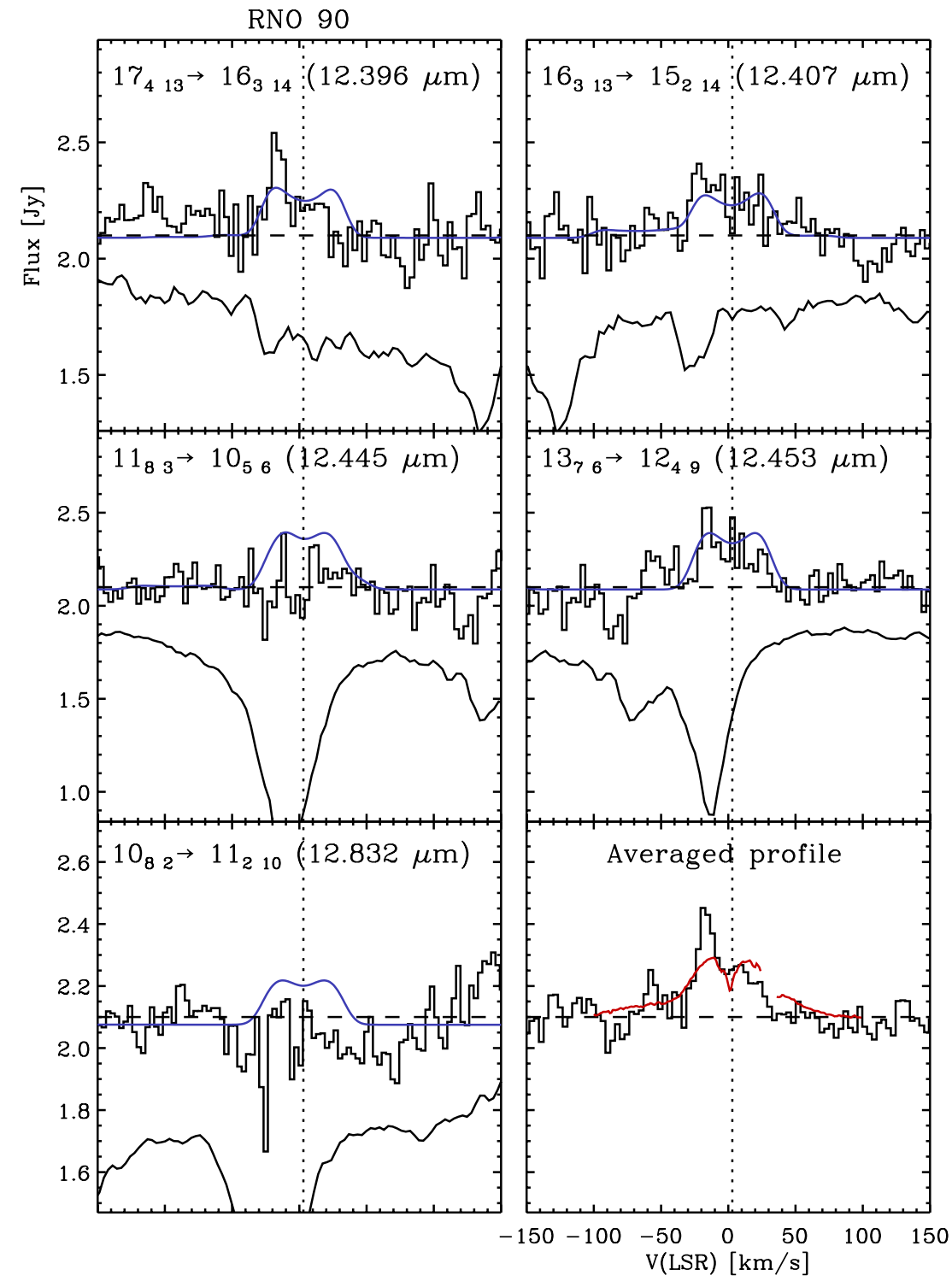

Figure 2. Same as Figure 1, but for RNO 90. The lower right panel presents the average of the three detected lines. The asymmetry visible as an increase in flux on the blue side of the detected lines is possibly real, but since it coincides with the telluric water lines, we are hesitant to discuss it in detail without further observations, preferably with different reflex motion Doppler shifts.

(A color version of this figure is available in the online journal.)

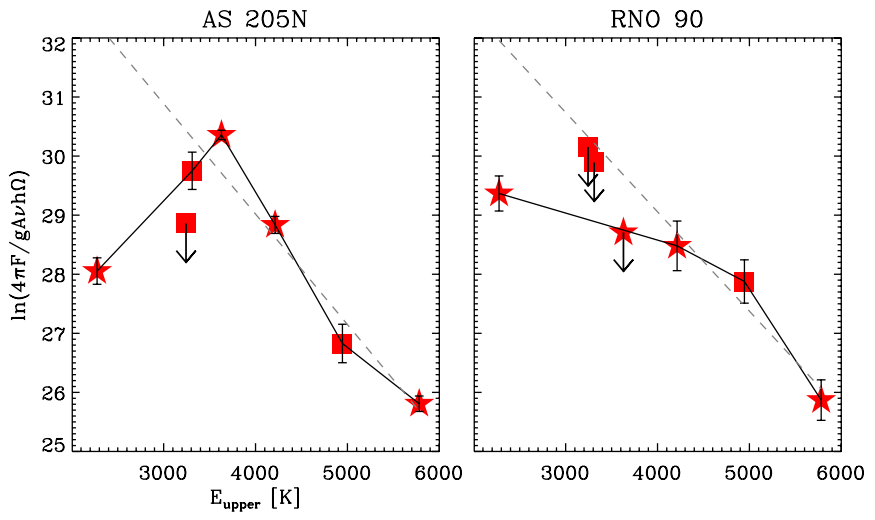

Figure 3. Rotation diagram for AS $205 \mathrm{~N}$ and RNO 90. The star symbols indicate ortho lines, while the square symbols indicate para lines. The points include a spin degeneracy of 3:1. The dashed lines are the best-fit single-temperature models (using the detected VISIR lines only). The units are cgs and the symbols have their usual meaning.

(A color version of this figure is available in the online journal.) line. Due to the higher luminosity of AS $205 \mathrm{~N}$, emitting radii span a wider range, here from 0.35 to $2.5 \mathrm{AU}\left(\sim 19 \mathrm{AU}^{2}\right)$. Using these measures, the column densities of water, averaged over the emitting radii, are $2 \times 10^{18}$ and $3 \times 10^{18} \mathrm{~cm}^{-2}$.

\section{DISCUSSION}

We have detected pure rotational lines from water vapor $\left(\mathrm{H}_{2}{ }^{16} \mathrm{O}\right)$ in protoplanetary disks around $\mathrm{T}$ Tauri stars from the ground using transitions in the atmospheric $N$-band window. Gas temperatures of 500-600 K are found, while the line widths of $30-60 \mathrm{~km} \mathrm{~s}^{-1}$ confirm that the disk surface at radii of $0.4-2.5 \mathrm{AU}$ is forming the lines. The resolved lines enable a much more direct determination of the emitting area and hence the column density of the water, as compared to that possible for spectrally unresolved Spitzer observations. We find that the assumptions used for the analysis of the Spitzer data generally hold, i.e., that the lines are formed in the surface of Keplerian disks at radii of $\sim 1 \mathrm{AU}$. The water column densities are found to be a little higher for AS $205 \mathrm{~N}$ than those determined by Salyk et al. (2008) 


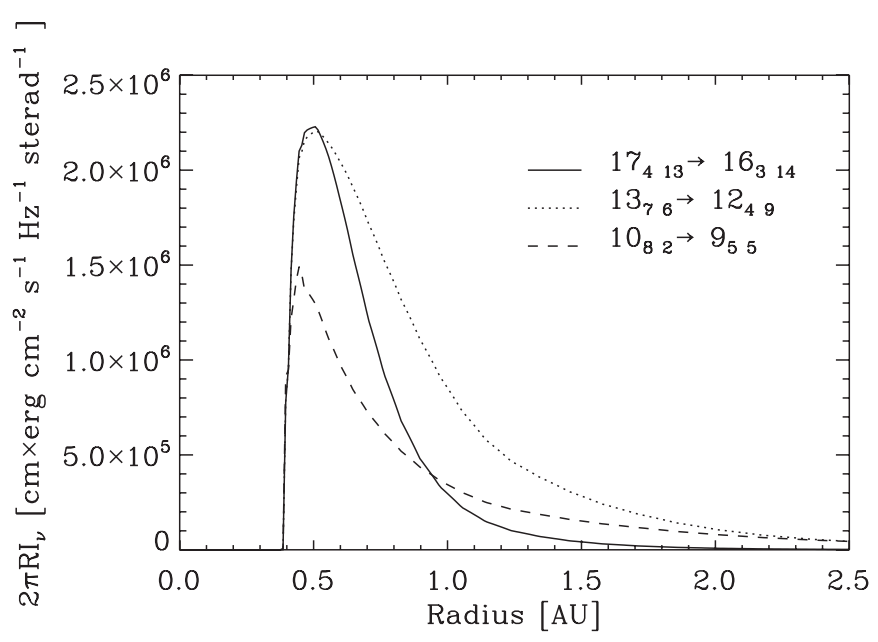

Figure 4. Continuum-subtracted surface brightness profiles of three lines for the RNO 90 model.

using rotational lines at somewhat longer wavelengths and lower energies. This appears to be consistent with the rotation diagram that indicates that lower excitation lines are less bright than expected for LTE level populations. This effect was also noted by Meijerink et al. (2009), who interpreted it as a depletion of water beyond $\sim 1 \mathrm{AU}$. More extensive $N$-band studies will help to further constrain the shape of the water rotation diagram to constrain the excitation and spatial distribution of water vapor in the inner regions of protoplanetary disks.

The derived water abundance is close to that predicted by Bethell \& Bergin (2009), based on a chemical model that includes water self-shielding. The two-dimensional RADLite models of both disks require high water abundances and high gas-to-dust ratios in order to reproduce the observed lines-a significant fraction of the available oxygen must be locked up in water vapor, and substantial dust settling to the disk midplane appears to have already taken place. An initial estimate of the $\mathrm{O} / \mathrm{P}$ ratio of the water suggests a value consistent with a high temperature equilibrium $(\mathrm{O} / \mathrm{P}=3)$, but higher signal-to-noise observations of more lines are required to confirm a high $\mathrm{O} / \mathrm{P}$ ratio. If confirmed, this is indicative of water formed via hot gas-phase chemistry, as opposed to water initially formed as ice in the outer disk and transported inward as part of migrating solids.

The spectra demonstrate that it is possible to obtain highresolution spectra of individual rotational lines of water in protoplanetary disks from the ground, in spite of telluric water absorption. These new tracers of the warm molecular layer have several unique advantages. The excitation energies and collisional rates of the high rotational levels are very similar to those of the ubiquitous fundamental rovibrational band of
$\mathrm{CO}$ at $4.7 \mu \mathrm{m}$. Given the chemical stability of $\mathrm{CO}$, this allows for detailed studies of the relative water abundances in a welldefined location in the disk surface. The lines are strong, also relative to the continuum, and their analysis is not hampered by strong photospheric lines from the central star-in contrast to the weaker, much higher energy water lines in the $3 \mu \mathrm{m}$ "hot band" (Salyk et al. 2008). The $N$-band lines may provide our only opportunity to obtain detailed velocity information of water at $\sim 1-10 \mathrm{AU}$ in disks around young solar analogs for the foreseeable future. Their observability from the ground also makes them available to the very high spatial resolution line imaging of $10 \mathrm{~m}$ class telescopes, as well as the next generation of Extremely Large Telescopes. As such, they will be important complements to the lower resolution spectroscopy of the James Webb Space Telescope.

Based on observations made at the ESO Paranal Observatory under program IDs 084.C-0635 and 179.C-0151. The authors acknowledge valuable discussions with Alain Smette.

\section{REFERENCES}

Andrews, S. M., Wilner, D. J., Hughes, A. M., Qi, C., \& Dullemond, C. P. 2009, ApJ, 700, 1502

Bethell, T., \& Bergin, E. 2009, Science, 326, 1675

Blake, G. A., \& Boogert, A. C. A. 2004, ApJ, 606, L73

Brittain, S. D., Simon, T., Najita, J. R., \& Rettig, T. W. 2007, ApJ, 659, 685

Carr, J. S., \& Najita, J. R. 2008, Science, 319, 1504

Carr, J. S., Tokunaga, A. T., \& Najita, J. 2004, ApJ, 603, 213

Chen, H., Myers, P. C., Ladd, E. F., \& Wood, D. O. S. 1995, ApJ, 445, 377

Crovisier, J., Leech, K., Bockelee-Morvan, D., Brooke, T. Y., Hanner, M. S., Altieri, B., Keller, H. U., \& Lellouch, E. 1997, Science, 275, 1904

Dullemond, C. P., Dominik, C., \& Natta, A. 2001, ApJ, 560, 957

Horne, K. 1986, PASP, 98, 609

Kawakita, H., Watanabe, J., Furusho, R., Fuse, T., Capria, M. T., De Sanctis, M. C., \& Cremonese, G. 2004, ApJ, 601, 1152

Lagage, P. O., et al. 2004, Messenger, 117, 12

Loinard, L., Torres, R. M., Mioduszewski, A. J., \& Rodríguez, L. F. 2008, ApJ, 675, L29

Meijerink, R., Pontoppidan, K. M., Blake, G. A., Poelman, D. R., \& Dullemond, C. P. 2009, ApJ, 704, 1471

Najita, J., Carr, J. S., \& Mathieu, R. D. 2003, ApJ, 589, 931

Pascucci, I., Apai, D., Luhman, K., Henning, T., Bouwman, J., Meyer, M. R., Lahuis, F., \& Natta, A. 2009, ApJ, 696, 143

Pontoppidan, K. M., Blake, G. A., van Dishoeck, E. F., Smette, A., Ireland, M. J., \& Brown, J. 2008, ApJ, 684, 1323

Pontoppidan, K. M., Meijerink, R., Dullemond, C. P., \& Blake, G. A. 2009, ApJ, 704, 1482

Pontoppidan, K. M., Salyk, C., Blake, G. A., Meijerink, R., Carr, J. S., \& Najita, J. 2010a, ApJ, 720, 887

Pontoppidan, K. M., et al. 2010b, ApJ, submitted

Rothman, L. S., et al. 2005, J. Quant. Spectrosc. Radiat. Transfer, 96, 139

Salyk, C., Blake, G. A., Boogert, A. C. A., \& Brown, J. M. 2009, ApJ, 699, 330

Salyk, C., Pontoppidan, K. M., Blake, G. A., Lahuis, F., van Dishoeck, E. F., \& Evans, N. J., II 2008, ApJ, 676, L49

Siess, L., Forestini, M., \& Bertout, C. 1997, A\&A, 326, 1001 